\title{
THE NEWBORN RESPIRATORY SYSTEM - PROGRAMMED TO RESPOND TO VARIABILITY?
}

\section{J.J. Pillow}

Centre for Neonatal Research and Education, University of Western Australia, Perth, WA, Australia

Promotion of lung volume recruitment in atelectatic lung and maintenance of existing recruited lung are vital goals of contemporary ventilatory support. In the mature adult lung, the recruitment of terminal airspaces are governed by power-law distributions, arising from avalanches associated with threshold pressure phenomena propagating down a branching tree structure. Superimposition of noise on the pressure waveform during conventional can promote recruitment of collapsed lung zones when the peak inspiratory pressure is at or around the lower inflexure and that this approach may also promote increased secretion of endogenous surfactant. The mechanism involves the phenomenon of stochastic resonance, which is most simply described as the addition of noise to a weak input signal to enhance output in a nonlinear dynamic system. It is a widespread, naturally occurring phenomenon that can be seen reflected in the patterns of world weather, fluctuations on the stock market, population biology, and optimal functioning of neural networks, to name but a few. Recent studies in newborn lambs show that incorporating variability in the breathing pattern enhances gas exchange, lung mechanics and regional ventilation distribution, indicating that these same stochastic principles apply to the immature lung. Further, increased expression of endogenous surfactant protein mRNA in lambs receiving variable volume ventilation compared to controlled mechanical ventilation suggests the fetus is programmed to respond to variable input signals. 